\title{
均匀和线性啁啾光纤布拉格光栅对光栅耦合器型 上下话路滤波器的下话路带宽的影响
}

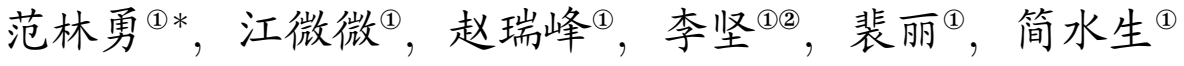 \\ (1) 北京交通大学光波技术研究所, 北京 100044 \\ (2) 铁道科学研究院通信信号研究所, 北京 100081 \\ * 通信作者. E-mail: skillfan@163.com
}

收稿日期: 2010-09-19; 接受日期: 2011-06-20

国家重点基础研究发展计划 (批准号: 2010CB328206)、国家高技术研究发展计划 (批准号: 2008AA01Z215) 和国家自然科学基金 (批准号: 60837002) 资助项目

\begin{abstract}
摘要 在光纤耦合器的耦合区写入光纤布拉格光栅是实现全光纤型上下话路滤波器的一种有效方 法. 为了分析将均匀光纤布拉格光栅和线性啁啾光纤布拉格光栅分别写入光纤耦合器的耦合区时, 对光纤光栅辅助失配耦合器型上下话路滤波器的下话路带宽的影响, 采用波长为 $248 \mathrm{~nm}$ 的紫外光, 在由标准单模光纤和高掺锗光敏光纤熔融拉锥制作的 $2 \times 2$ 光纤失配耦合器上分别写入均匀光纤布 拉格光栅和线性啁啾光纤布拉格光栅, 实验制作了均匀光纤布拉格光栅辅助失配耦合器型上下话路 滤波器和线性啁啾光纤布拉格光栅辅助失配耦合器型上下话路滤波器. 经测试, 在下话路端口, 前 者的 $3 \mathrm{~dB}$ 带宽和 $20 \mathrm{~dB}$ 带宽分别约为 $0.1 \mathrm{~nm}$ 和 $0.3 \mathrm{~nm}$; 后者的 $3 \mathrm{~dB}$ 带宽和 $20 \mathrm{~dB}$ 带宽分别约为 $0.8 \mathrm{~nm}$ 和 $2 \mathrm{~nm}$. 实验结果与理论分析具有较好的一致性, 这说明可以通过调节在光纤失配耦合器的 耦合区的均匀部分写入的线性啁啾光纤布拉格光栅的啁啾量来调节所制器件的下话路带宽.
\end{abstract}

\section{关键词 光上下话路滤波器 光纤布拉格光栅 耦合器 光纤通信 光器件}

\section{1 引言}

光上下话路滤波器是密集波分复用 (DWDM, dense wavelength devision multiplexing) 系统的重要 器件. 目前光上下话路滤波器主要有阵列波导光栅 (AWG, arrayed waveguide grating) 型和光纤光栅型 等. 阵列波导光栅型光上下话路器的难点在于制作波导光栅, 即控制玻璃膜的厚度、成分与缺欠等工 艺 ${ }^{[1]}$. 光纤布拉格光柾 (FBG, fiber Bragg grating) 有着良好的复用和解复用的特性. 然而, 只有两个 端口和只能工作于反射模式是它的缺点, 而且常需要配合其他器件一块使用, 比如光环形器等 ${ }^{[2]}$. 因 此, 基于光纤布拉格光栅和光环形器的光插分复用器 (OADM, optical add-drop multiplexer) 的插入损 耗较高, 体积较大, 成本较高, 集成较难 ${ }^{[3]}$. 光纤布拉格光栅耦合器将光纤布拉格光栅写入耦合器的 耦合区, 集合了耦合器和光栅的特点. 基于光纤布拉格光栅耦合器的 OADM 具有四端口、低损耗、低 成本和紧凑性好等优点. 光纤布拉格光栅耦合器型上下话路滤波器主要有 3 种: 光纤布拉格光栅反射 型、光纤布拉格光栅破坏型和光纤布拉格光栅辅助失配型. 研究人员制作出了耦合效率、 $3 \mathrm{~dB}$ 带宽、 
插入损耗和回波损耗分别约为 $99 \% 、 1.2 \mathrm{~nm} 、 7 \mathrm{~dB}$ 和 $-30 \mathrm{~dB}$ 的光纤布拉格光栅反射型 $\mathrm{OADM}^{[4]}$. 对 于光纤布拉格光栅破坏型, 在实验上已制作出线宽为 $0.7 \mathrm{~nm}$, 下载中心波长为 $1535 \mathrm{~nm}$, 峰值透射率 为 $70 \%$, 隔离度为 $13 \mathrm{~dB}$ 的下话路滤波器 ${ }^{[5]}$. 基于光纤布拉格光栅辅助失配耦合器型的上下话路滤波 器, 实验上制作出了峰值反射率和 $3 \mathrm{~dB}$ 带宽分别约为 $20 \mathrm{~dB}$ 和 $0.08 \mathrm{~nm}$ 的滤波器 ${ }^{[6]}$. 与第 3 种类型 的滤波器相比, 前两种滤波器的带宽较宽. 但是前两种滤波器属于干涉型, 较难制作, 而第 3 种滤波器 是非干涉型的, 较容易制作. 较窄的带宽会限制下话路端口的通信容量. 因此, 制作出宽带宽的光纤布 拉格光栅辅助失配耦合器型上下话路滤波器是很必要的. 文献 [7] 采用在光纤失配耦合器的耦合区的 非均匀部分写入均匀 FBG 的方法, 实验制作了最大反射率约为 $20 \mathrm{~dB}, 3 \mathrm{~dB}$ 带宽约为 $2 \mathrm{~nm}$ 的光纤布 拉格光栅辅助失配耦合器型的上下话路滤波器, 并推测在失配耦合器的耦合区的均匀部分写入啁啾光 纤光栅也可以增加该器件下话路带宽的结论.

本文在文献 [7] 所推测出的结论的基础上, 从理论和实验两方面, 分别研究两种光纤布拉格光栅 辅助失配耦合器型上下话路滤波器, 一种在失配耦合器的耦合区的均匀部分中写入均匀光纤布拉格光 栅, 另一种在失配耦合器的耦合区的均匀部分写入线性啁啾光纤布拉格光栅. 分别测试并比较所制器 件的 $3 \mathrm{~dB}$ 和 $20 \mathrm{~dB}$ 带宽, 分析均匀光纤布拉格光栅和线性啁啾光纤布拉格光栅对所制器件下话路带 宽的影响, 分析发现后者的带宽大于前者的带宽.

\section{2 原理分析}

光纤布拉格光栅辅助失配型上下话路滤波器的基本结构如图 1 所示. 首先用两根差异较大的光 纤制成不匹配耦合器, 然后在耦合区中的一个纤芯上写入布拉格光栅. 由于两纤芯失配, 在无光栅区, 两纤心之间的耦合作用十分小, 主要起传导光功率的作用. 在光椓区, 由于单芯写入光栅, 实现了正反 向基模的功率转化. 如图 1 所示, 在端口 1 输入光信号, 根据布拉格光俩的作用建立耦合, 满足条件

$$
{ }^{1} \beta_{01}\left(\lambda_{12}\right)+{ }^{2} \beta_{01}\left(\lambda_{12}\right)=K=2 \pi / \Lambda
$$

的信号 $\lambda_{12}$ 将从端口 4 输出. (1) 式中, ${ }^{1} \beta_{01}$ 和 ${ }^{2} \beta_{01}$ 分别为纤芯 1 和纤芯 2 中 $\mathrm{LP}_{01}$ 模的传输常数, $\lambda_{12}$ 为光栅耦合器的反射波长, $K$ 为光栅的波数, $\Lambda$ 为光栅的周期. 除了波长为 $\lambda_{12}$ 的信号外, 其他波 长的信号继续在纤芯 1 中传输, 并从端口 2 输出. 于是, 该滤波器实现了下话路的功能. 当在端口 3 输 入波长为 $\lambda_{12}$ 的信号时, 该信号将与纤芯 1 中的信号一同从端口 2 输出, 于是实现了上话路的功能 ${ }^{[3]}$. 端口 1 、端口 2 、端口 3 和端口 4 分别用作输入端口、输出端口、上话路端口和下话路端口.

该器件各端口的输出特性可由如下耦合模方程表达:

$$
\frac{\mathrm{d}}{\mathrm{d} z}\left[\begin{array}{l}
g_{1+} \\
g_{1-} \\
g_{2+} \\
g_{2-}
\end{array}\right]=j \frac{1}{1-N_{12}^{2}}\left[\begin{array}{cccc}
Q_{11} & Q_{12} & Q_{13} & Q_{14} \\
Q_{21} & Q_{22} & Q_{23} & Q_{24} \\
Q_{31} & Q_{32} & Q_{33} & Q_{34} \\
Q_{41} & Q_{42} & Q_{43} & Q_{44}
\end{array}\right]\left[\begin{array}{l}
g_{1+} \\
g_{1-} \\
g_{2+} \\
g_{2-}
\end{array}\right],
$$

(2) 式中, $g_{i+}, g_{i-}$ 为纤芯 $i(i=1,2)$ 中前后向 $\mathrm{LP}_{01}$ 模的慢变振幅, $N_{12}$ 和 $Q_{m n}(m=1,2,3,4 ; n=1$, $2,3,4)$ 为与两根光纤参量相关的系数. 


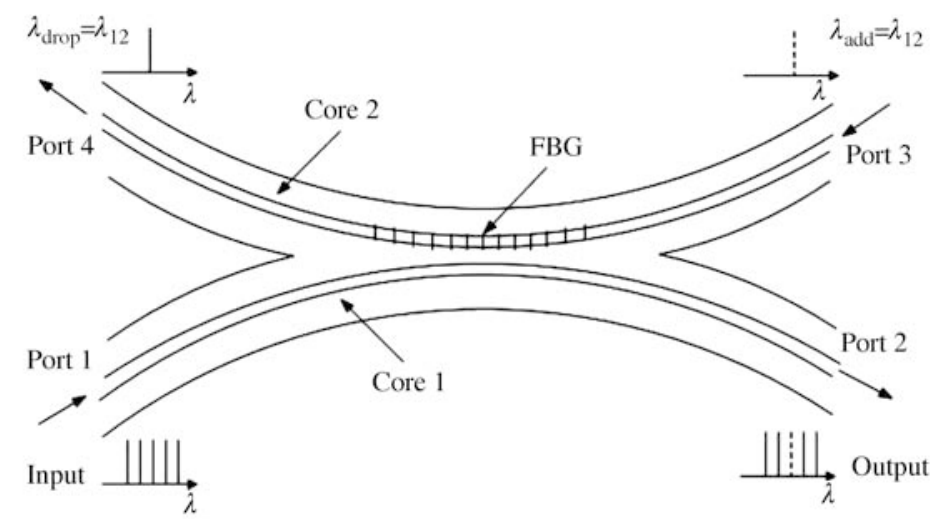

图 1 光纤布拉格光栅辅助失配型上下话路滤波器示意图

Figure 1 Configuration of the Bragg grating assisted mismatched coupler for add/drop filter

当在耦合器的耦合区的纤芯 2 中写入均匀光纤布拉格光栅时, 耦合区的折射率调制系数可以表 示为

$$
\Delta n(z)=\Delta n_{20}+\delta n_{2} f(z) \cos \left(\frac{2 \pi}{\Lambda} z\right),
$$

(3) 式中, $\Delta n_{20} 、 \delta n_{2}$ 和 $f(z)$ 分别为在光栅的一个空间周期上的平均折射率变化的直流分量、折射率 变化的交流分量和切趾函数. 当在耦合器的耦合区的纤芯 2 中写入线性啁啾光纤布拉格光栅时, 耦合 区的折射率调制系数可以表示为

$$
\Delta n(z)=\Delta n_{20}+\delta n_{2} f(z) \cos \left(\frac{2 \pi}{\Lambda_{c}} z+2 \int_{0}^{z} \phi(\xi) \mathrm{d} \xi\right),
$$

(4) 式中, $\phi(z)=(2 \pi C z) /\left(\Lambda_{c} L\right)$, 其中 $\Lambda_{c} 、 C$ 和 $L$ 分别为线性啁啾光纤布拉格光栅的中心波长、啁啾 量和光栅长度.

假设光栅长度为 $L$, 当一束光从端口 1 射入时, (2) 式的边界条件可以表示为

$$
g_{1+}(0)=1, \quad g_{1-}(L)=0, \quad g_{2+}(0)=0, \quad g_{2-}(L)=0 .
$$

采用 Broyden 迭代法和 Runge-Kutta 法可以计算出 (2) 式中各个端口的输出功率. 选用如下参数进行 计算 $r_{1}=4 \mu \mathrm{m}, r_{2}=3 \mu \mathrm{m}, d=10 \mu \mathrm{m}, n_{1}=1.449, n_{c}=1.4442, L=40 \mathrm{~mm}, \Delta n_{1}=\Delta n_{2}=0.48 \%$, $\Delta n_{10}=\Delta n_{20}=0, \delta n_{1}=0, \delta n_{2}=5 \times 10^{-4}, L_{c}=L, C=2 \times 10^{-5}\left(r_{1}, r_{2}, d, n_{c}, \Delta n_{1}, \Delta n_{2}, \delta n_{1}\right.$ 和 $L_{c}$ 分别为纤芯 1 和纤芯 2 的半径, 纤芯 1 和纤芯 2 之间的距离, 包层的折射率, 纤芯 1 的折射率差, 纤 芯 2 的折射率差, 纤芯 1 的交流分量和耦合区的长度). 数值模拟结果如图 2 所示.

图 2 是在所设计的上下话路器的端口 1 输入光信号, 光信号经过该器件后各个端口的输出谱. 在 光柱反射带宽内, 端口 4 的归一化功率几乎达到 $0 \mathrm{~dB}$, 端口 1 的归一化功率从 $0 \mathrm{~dB}$ 下降到 $-13 \mathrm{~dB}$, 端 口 2 和端口 3 几乎没有功率输出. 由此可见, 从端口 1 输入的在光棶反射带宽内的光信号几乎 $100 \%$ 的 从器件的端口 4 输出, 实现了下话路功能; 在光栅反射带宽外, 端口 2 的归一化功率几乎达到 $0 \mathrm{~dB}$, 而其他端口的功率都比较小, 由此可见端口 2 是该器件的输出端. 图 2 中, 在整个波长范围内, 端口 1 和端口 4 , 端口 2 和端口 3 的功率差均约为 $13 \mathrm{~dB}$. 这可能是由于在耦合区两根光纤的纤芯距较小, 两 个纤芯的折射率不同, 两个纤芯的模式发生不完全耦合, 使得纤芯 2 中向端口 4 传输的光一小部分耦 合到纤芯 1 , 并从端口 1 输出, 也使得纤芯 1 中向端口 2 传输的光一小部分耦合到纤芯 2 , 并从端口 3 

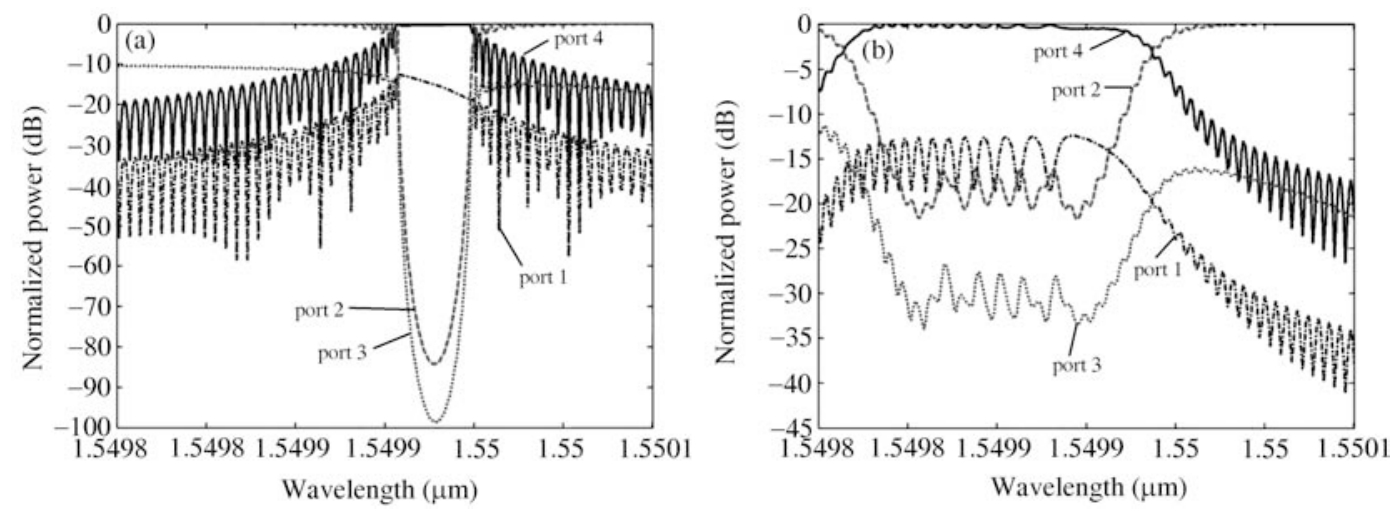

图 2 当入射光从端口 1 输入时, 各个端口的输出功率

Figure 2 Outputs from the four ports when light is injected into port 1. (a) OADF using a uniform FBG-assisted coupler; (b) OADF using a linearly chirped FBG-assisted coupler

输出. 计算中设定耦合区两光纤间的纤芯距恒定, 所以两纤芯间的耦合率恒定, 于是端口 1 和端口 4 , 端口 2 和端口 3 的功率差恒定. 由图 2 可知, 器件的插入损耗约为 0 , 回波损耗约为 $-13 \mathrm{~dB}$. 回波损 耗可能主要是由于在耦合区纤芯 2 中向端口 4 传输的光一小部分耦合到纤芯 1 , 并从端口 1 输出而引 起的. 如要实现较小的回波损耗, 可以增加两个纤芯间的有效折射率差, 降低纤芯 1 和纤芯 2 之间的 耦合率.

如图 2(a) 所示, 当将均匀的 FBG 写入耦合器时, 器件下话路端口 (port 4) 的 $3 \mathrm{~dB}$ 和 $20 \mathrm{~dB}$ 带 宽分别为 $0.05 \mathrm{~nm}$ 和 $0.07 \mathrm{~nm}$. 如图 2(b) 所示, 当将线性啁啾的 $\mathrm{FBG}$ 写入耦合器时, 器件下话路端 口 (port 4) 的 $3 \mathrm{~dB}$ 和 $20 \mathrm{~dB}$ 带宽分别为 $0.17 \mathrm{~nm}$ 和 $0.30 \mathrm{~nm}$. 由此可见, 当在耦合器上写入线性啁啾 的 FBG 时可以增加该器件下话路端口的带宽. 比较图 2(a) 和图 2(b), 可以发现图 2(b) 所示器件的中 心波长向短波长方向漂移了 $0.07 \mathrm{~nm}$, 各个端口的带内功率曲线都有振荡现象, 器件下话路端口 (port 4) 的带内平坦度约为 $\pm 0.5 \mathrm{~dB}$. 容易发现, 端口 1 和端口 4 , 端口 2 和端口 3 呈现出对应的正弦和余 弦的变化规律. 这可能是两纤芯间的功率耦合引起的, 因此可以通过增加两个纤芯间的有效折射率差, 降低纤芯 1 和纤芯 2 之间的耦合率, 以消除带内的功率振荡.

\section{3 实验结果与分析}

分别取一根标准单模光纤和一根本实验室自制的高掺锗光敏光纤, 采用熔融拉锥法制作失配耦合 器. 在相同条件下, 制得两个耦合区均匀部分长度约为 $40 \mathrm{~mm}$ 的失配耦合器. 采用相位掩膜法, 在其 中一个耦合器的耦合区的均匀部分写入长度为 $40 \mathrm{~mm}$, 周期为 $1075 \mathrm{~nm}$ 的均匀光纤布拉格光栅. 在另 一个耦合器的耦合区的均匀部分写入长度为 $40 \mathrm{~mm}$, 中心波长为 $1073.07 \mathrm{~nm}$, 啁啾量为 0.68 的线性 啁啾光纤布拉格光栅. 使用的 $\mathrm{KeF}$ 准分子激光器的波长、频率和能量分别为 $248 \mathrm{~nm} 、 8 \mathrm{~Hz}$ 和 $80 \mathrm{~mJ}$. 制得了基于均匀光纤布拉格光栅的辅助失配耦合器型上下话路滤波器和基于线性啁啾光纤布拉格光 栅的辅助失配耦合器型上下话路滤波器, 分别命名为滤波器 1 和滤波器 2 .

从端口 1 输入一宽带光源. 用 ANDO 公司的光谱分析仪 $\mathrm{AQ6317C}$ (分辨率: $0.01 \mathrm{~nm}$ ) 分别检测端 口 1 和端口 4 的输出信号. 如图 3(a) 所示, 滤波器 1 的交叉耦合发生在 $\lambda_{12}=1541.9 \mathrm{~nm}$ 处, 端口 4 处 信号的耦合效率、 $3 \mathrm{~dB}$ 和 $20 \mathrm{~dB}$ 带宽分别约为 $99 \% 、 0.1 \mathrm{~nm}$ 和 $0.3 \mathrm{~nm}$; 如图 $3(\mathrm{~b})$ 所示, 滤波器 2 的 

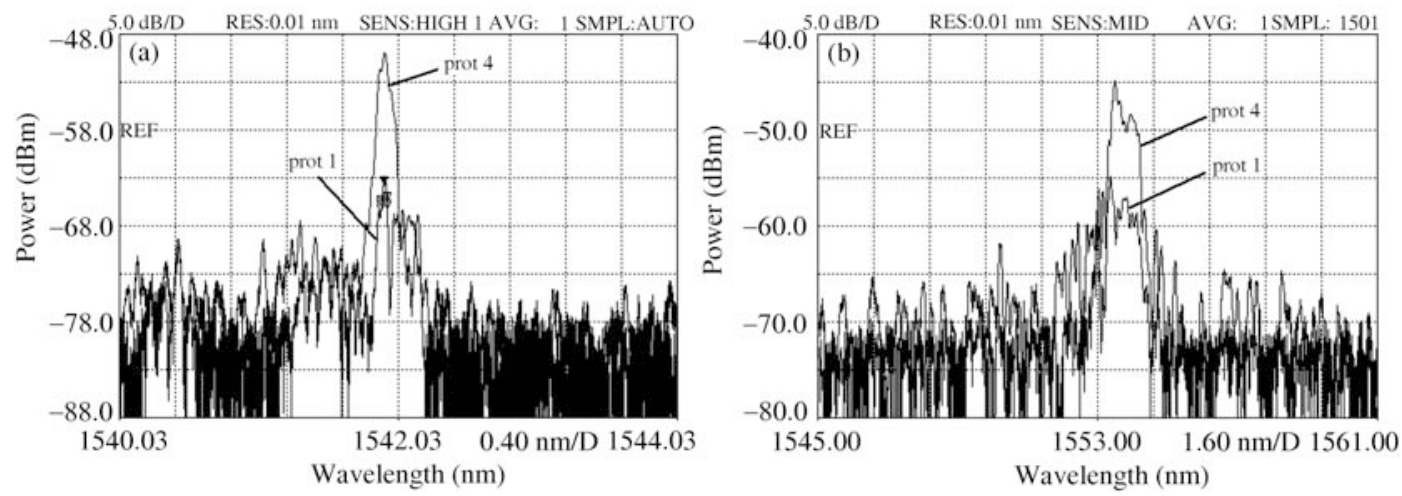

图 3 端口 1 输入宽带光源时, 端口 1 和端口 4 的输出谱

Figure 3 Outputs from port 1 and port 4 when a broadband light was injected into port 1. (a) The OADF using a uniform FBG-assisted coupler; (b) the OADF using a linearly chirped FBG-assisted coupler

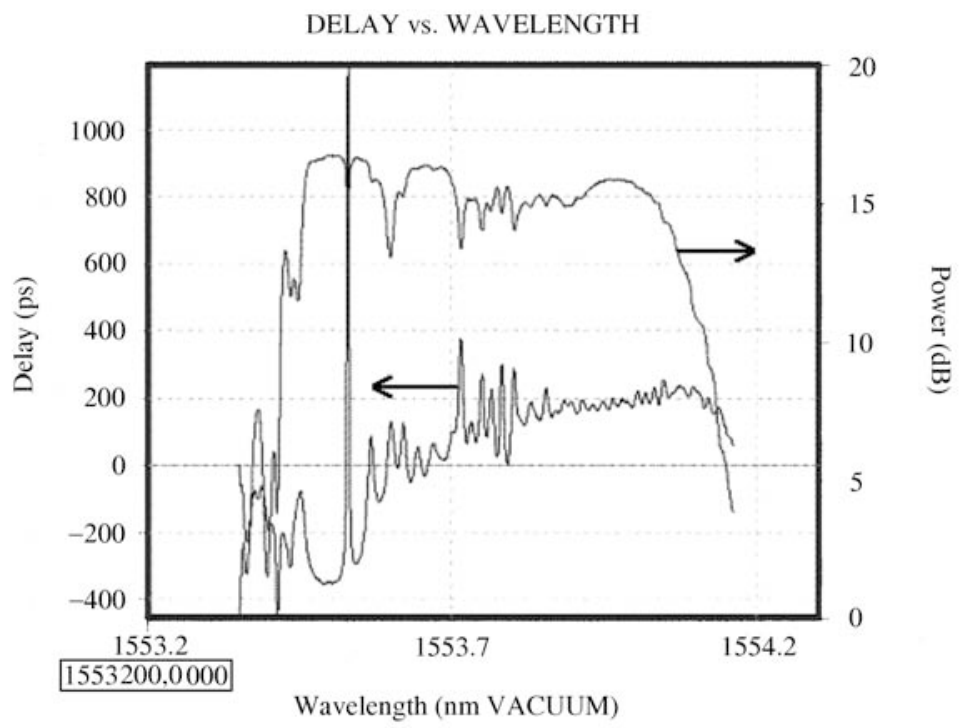

图 4 基于线性啁啾光纤布拉格光栅的辅助失配耦合器型上下话路滤波器的下话路输出光谱和时延特性测试图

Figure 4 Time delay and output from port 4 of the OADF using a linearly chirped FBG-assisted coupler

交叉耦合发生在 $\lambda_{12}=1553.8 \mathrm{~nm}$ 处, 端口 4 处信号的耦合效率、 $3 \mathrm{~dB}$ 和 $20 \mathrm{~dB}$ 带宽分别约为 $99 \% 、 0.8$ $\mathrm{nm}$ 和 $2 \mathrm{~nm}$. 滤波器 2 的带宽明显大于滤波器 1 的. 从实验结果可知, 通过在失配耦合器的耦合区的 均匀部分写入线性啁啾光纤布拉格光栅, 可以增大滤波器下话路端口的带宽. 这里滤波器 2 的 $3 \mathrm{~dB}$ 带宽小于文献 [7] 中所制器件的 $2 \mathrm{~nm}$. 然而, 采用不同啁啾量的线性啁啾布拉格光栅可以得到不同的 带宽, 也就是说可以达到一个定量的调节, 并且可能具有较好的重复性. 比较图 3(a) 与图 4(b), 图 4(b) 所示器件 (滤波器 2 ) 的端口 4 的输出谱出现带内功率振荡, 这与前边的理论计算结果相一致.

为了进一步检测滤波器 2 的相关特性, 采用 PerkinElmer 公司的 CD400 色散分析仪 (分辨率: $0.001 \mathrm{~nm}$ ) 对滤波器 2 的下话路输出进行色散测试, 结果如图 4 所示. 由图可见, 所制器件的带内平坦 度约为 $\pm 2 \mathrm{~dB}$, 在 $1553.4 \mathrm{~nm}$ 到 $1554.2 \mathrm{~nm}$ 的波长范围内的时延差约为 $500 \mathrm{ps}$. 

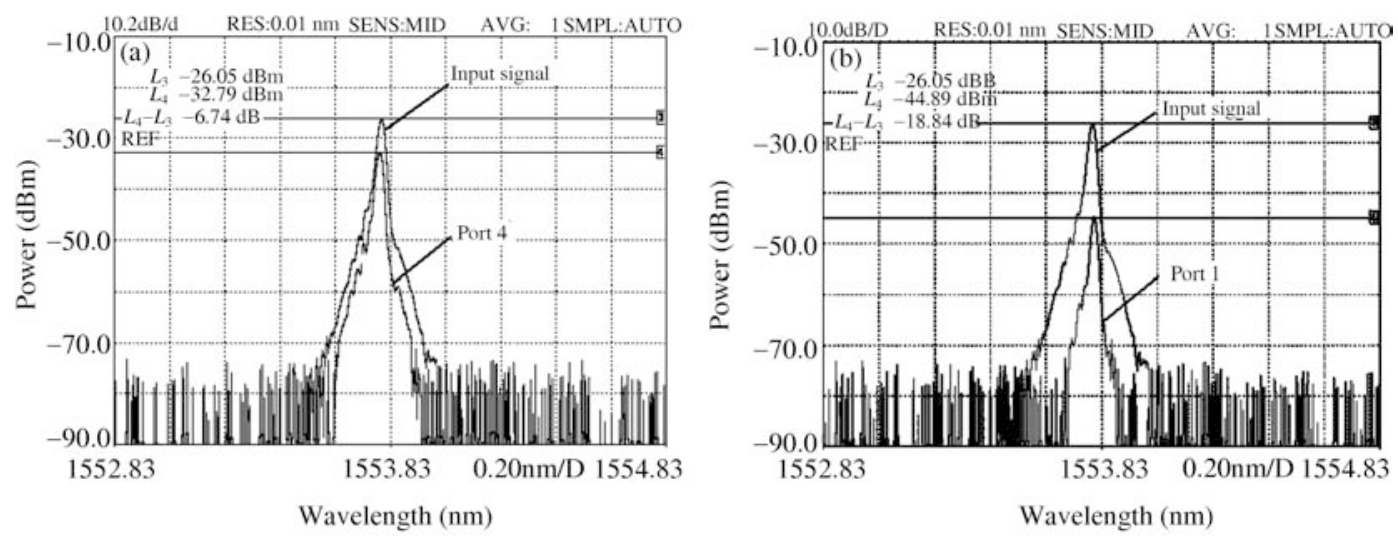

图 5 端口 1 输入一信号 $\left(\lambda_{12}=1553.8 \mathrm{~nm}\right)$ 时, (a) 端口 4 的输出谱; (b) 端口 1 的输出谱

Figure 5 (a) Output from port 4; (b) output from port 1, with an input ( $\lambda_{12}=1553.8 \mathrm{~nm}$ ) to port 1

根据图 4 的结果, 将一信号 $\left(\lambda_{12}=1553.8 \mathrm{~nm}\right)$ 由端口 1 输入. 用 ANDO 公司的光谱分析仪 $\mathrm{AQ} 6317 \mathrm{C}$ (分辨率: $0.01 \mathrm{~nm}$ ) 分别检测端口 1 和端口 4 的输出信号. 如图 5 所示, 滤波器 2 的插入 损耗约为 $6.74 \mathrm{~dB}$, 回波损耗约为 $-18.84 \mathrm{~dB}$. 在用熔融拉雉法制作光纤耦合器时, 耦合器的雉区和耦 合区都变得比较细, 纤芯变细, 部分光扩散到了包层中, 然后以消逝场的形式泄露到空气中, 于是导致 插入损耗. 在纤芯 2 的光栅的作用下, 下话路信号向端口 4 方向传输. 在耦合区两根光纤的纤芯距较 小, 两个纤芯的折射率不同, 两个纤芯的模式发生不完全耦合, 使得纤芯 2 中向端口 4 传输的光一小 部分耦合到纤芯 1 , 并从端口 1 输出, 于是导致了器件的回波损耗. 从图 3 和图 5 可见, 实验中, 在整 个波长范围内, 两个滤波器的端口 4 的功率高于端口 1 的功率, 存在约 $12 \mathrm{~dB}$ 的差值. 这与第一部分 的理论分析相一致. 从以上分析可知, 可以通过进一步优化所制光纤耦合器的参数, 如两个纤芯间的 有效折射率差、耦合区长度、纤芯 1 和纤芯 2 之间的距离等, 减少光以辐射模的形式产生的泄露损耗, 降低耦合区中两纤芯间的耦合率以减小回波损耗, 从而制得低插入损耗和低回波损耗的器件.

\section{4 总结}

用耦合模理论分析了均匀光纤布拉格光栅和线性啁啾光纤布拉格光栅对光栅辅助失配耦合器型 上下话路滤波器的下话路带宽的影响. 采用熔融拉堆法, 在自行设计的耦合器制作平台上, 用一根标 准单模光纤和一根自制的高掺锗光敏光纤制成失配耦合器. 通过在失配耦合器的耦合区的均匀部分分 别写入均匀光纤布拉格光栅和线性啁啾光纤布拉格光栅, 实验制得两种不同的上下话路滤波器. 在下 话路端口, 前者的 $3 \mathrm{~dB}$ 带宽和 $20 \mathrm{~dB}$ 带宽分别约为 $0.1 \mathrm{~nm}$ 和 $0.3 \mathrm{~nm}$; 后者的 $3 \mathrm{~dB}$ 带宽和 $20 \mathrm{~dB}$ 带 宽分别约为 $0.8 \mathrm{~nm}$ 和 $2 \mathrm{~nm}$. 实验结果与理论分析较一致. 这表明通过调节在光纤失配耦合器的耦合 区的均匀部分写入的线性啁啾光纤布拉格光栅的啁啾量可以调节该器件的下话路带宽.

\section{参考文献}

1 Pei L, Zhao R F, Ning T G, et al. Wavelength-division demultiplexer based on FBG coupler. Acta Opt Sin, 2009, 29: 308-311 [裴丽, 赵瑞峰, 宁提纲, 等. 基于光纤光栅辅助耦合的 WDM 下话路研究. 光学学报, 2009, 29: 308-311]

2 Dong L, Hua P, Birks T A, et al. Novel add/drop filters for wavelength-division multiplexing optical fiber systems using a Bragg grating assisted mismatched coupler. IEEE Photonic Tech L, 1996, 8: 1656-1658 
3 Riziotis C. Novel full-cycle-coupler-based optical add-drop multiplexer and performance characteristics at 40-Gb/s WDM networks. J Lightw Technol, 2003, 21: 1828-1837

4 Baumann I, Seifert J, Nowak W, et al. Compact all-fiber add-drop-multiplexer using fiber Bragg gratings. IEEE Photonic Tech L, 1996, 8: 1331-1333

5 Archambault J L, Russell P St J, Barcelos S, et al. Grating-frustrated coupler: a novel channel-dropping filter in single-mode optical fiber. Opt Lett, 1994, 19: 180-182

6 Dong X W, Pei L, Jian S S. Add/drop channel filter based on fiber-Bragg-grating-assisted coupler fabricated by asymmetric fused taper technology. Acta Phys Sin, 2006, 55: 4739-4743 [董小伟, 裴丽, 简水生. 非对称熔雉法制作光纤 光栅辅助耦合器型上下话路滤波器. 物理学报, 2006, 59: 4739-4743]

7 Fan L Y, Li J, Jiang W W, et al. Experimental fabrication of Bragg grating-assistedmismatched coupler for add/drop filters. Laser Optoelectron Prog, 2010, 47: 30603 [范林勇, 李坚, 江微微, 等. 光纤布拉格光栅辅助耦合器型上下话路滤波器 的实验制作. 激光与光电子学进展, 2010, 47: 30603]

\title{
Influences on the bandwidth at the drop port of the add/drop filter using a fiber grating coupler by uniform and linearly chirped fiber Bragg grating
}

\author{
FAN LinYong ${ }^{1 *}$, JIANG WeiWei ${ }^{1}$, ZHAO RuiFeng ${ }^{1}$, LI Jian ${ }^{1,2}$, PEI $\operatorname{Li}^{1} \&$ JIAN ShuiSheng ${ }^{1}$ \\ 1 Institute of Lightwave Technology, Beijing Jiaotong University, Beijing 100044, China; \\ 2 Signal \& Communicaiton Research Institute, China Academy of Railway Sciences, Beijing 100081, China \\ *E-mail: skillfan@163.com
}

\begin{abstract}
Writing a fiber Bragg grating (FBG) in an optical fiber coupler is a very effective way to implement an optical add/drop filter (OADF). In order to analyze the influences on the bandwidth at the drop port of the OADF using fiber grating-assisted mismatched coupler, a uniform FBG and a linearly chirped FBG were written on the coupler, and two different OADFs were fabricated. The fiber grating is written in a $2 \times 2$ mismatched coupler, which is fused and tapered with a standard single mode fiber (SMF) and a high germanium doped photosensitive fiber, by using a $248 \mathrm{~nm}$ ultraviolet laser. For the OADF using the uniform FBG, the $3 \mathrm{~dB}$ and $20 \mathrm{~dB}$ bandwidths are $0.1 \mathrm{~nm}$ and $0.3 \mathrm{~nm}$, respectively; for the OADF using the linear chirped FBG, the $3 \mathrm{~dB}$ and $20 \mathrm{~dB}$ bandwidths are $0.8 \mathrm{~nm}$ and $2 \mathrm{~nm}$, respectively. The experimental results meet the theoretical analysis well. The bandwidth at the drop port of the device can be adjusted by adjusting the chirped coefficient of the linearly chirped FBG.
\end{abstract}

Keywords optical add/drop filter, fiber Bragg grating, coupler, optical fiber communication, optical device

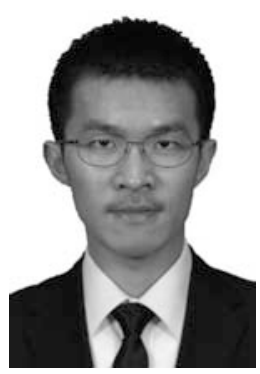

FAN LinYong was born in 1984. He received the Ph.D. degree in communication and imformation system from Beijing Jiaotong University, Beijing in 2012. Currently, he is a researcher at Huawei Technologies Company. His research interests include optical fiber communication, optical fiber sensing, and optical devices. 